Bull. Austral. Math. Soc.

Vol. 55 (1997) [161-168]

\title{
QUADRATIC APPROXIMATION OF SOLUTIONS FOR ORDINARY DIFFERENTIAL EQUATIONS
}

\author{
JuAN J. NiETo
}

\begin{abstract}
We present a generalisation of the quasilinearisation method to obtain a monotone sequence of approximate solutions that converges quadratically to a solution of a nonlinear ordinary differential equation of order $n \geqslant 1$.
\end{abstract}

\section{INTRODUCTION}

The method of quasilinearisation is a classical tool to obtain approximate solutions of nonlinear problems $[1,2]$. Under some suitable conditions, it is possible to construct a monotone increasing sequence of approximate solutions that converges quadratically to a solution. For instance, consider the following initial problem for a first order ordinary differential equation

$$
u^{\prime}(t)=f(u(t)), t \in I=[0, T], u(0)=u_{0} .
$$

If $f$ is convex, then we can write $f(u)=\max _{v} g(u, v)$ where $g(u, v)$ is linear in $u$. Then, starting with a reasonable initial approximation, one can generate a sequence of approximate solutions converging quadraticaly to the solution of (1).

Recently the method of quasilinearisation was generalised [4] to the case when $f(u)+M u^{2}$ is convex for some $M>0$.

On the other hand, it is well known that the method of lower and upper solutions coupled with the monotone iterative technique provides a practical tool to generate monotone sequences that converge to the maximal and minimal solutions [3]. However, as is shown in [5], the convergence of the monotone method is, in general, not quadratic.

The purpose of this paper is to extend the method of quasilinearisation to a nonlinear ordinary differential equation of order $n \geqslant 1$. Concretely, we study the following nonlinear boundary value problem

$$
[D u](t)=f(t, u(t)), t \in I=[0, T], B(u)=b
$$

Received 12th March, 1996

Research partially supported by D.G.I.C.Y.T. (Spain), project PB94 - 0610, and by EC Network, CHRX - CT94 - 0555.

Copyright Clearance Centre, Inc. Serial-fee code: 0004-9729/97 \$A2.00+0.00. 
where $D$ is a linear ordinary differential operator of order $n \geqslant 1$ defined by

$$
D u=u^{(n)}+\sum_{i=1}^{n-1} a_{i} u^{(i)}, a_{i} \in \mathbb{R}
$$

$f: I \times \mathbb{R} \rightarrow \mathbb{R}$ is continuous, $B(u)=\left(B_{i}(u)\right)_{i=0}^{n-1}, b=\left(b_{i}\right)_{i=0}^{n-1} \in \mathbb{R}^{n}$, and for any $i=0,1, \ldots, n-1, B_{i}: C^{n-1}(I) \rightarrow \mathbb{R}$ is linear.

The paper is organised as follows. In Section 2 we present some results on the linear differential operator $D: E \rightarrow C(I), E=\left\{u \in C^{n}(I): B(u)=0\right\}$, and recall the upper and lower solution method, as well as the monotone iterative technique, for the nonlinear problem (2).

Then, in Section 3, we develop the method of quasilinearisation for (2) provided that there exists $M>0$ such that $f(t, u)+M u^{2}$ is convex in $u$. Thus, we obtain a sequence of monotone increasing approximate solutions that converge quadratically to a solution of (2).

Finally, we apply the result to some particular situations for first, second, and higher order problems. Some examples include previous results and some other examples are new.

\section{Preliminaries}

Let $F$ be a normed space with norm \|\| , and $\left\{u_{k}\right\}$ a sequence in $F$. Suppose that $\left\{u_{k}\right\}$ converges to $u \in F$. We say that $\left\{u_{k}\right\}$ converges quadratically to $u \in F$ if there exist $k_{0} \in \mathbb{N}$, and $\lambda>0$ such that for $k \geqslant k_{0}$ we have that

$$
\left\|u_{k+1}-u\right\| \leqslant \lambda \cdot\left\|u_{k}-u\right\|^{2} .
$$

We now consider the homogeneous linear problem

$$
[D u](t)=0, t \in I=[0, T], B(u)=0 .
$$

If it has only the trivial solution, then the linear problem

$$
[D u](t)=h, t \in I=[0, T], B(u)=b
$$

has a unique solution $u$ for any $h \in C(I)$. Therefore (see, for instance, [9]), there exists a Green's function $G: I \times I \rightarrow \mathbb{R}$ such that the solution of (4) is given by

$$
u(t)=v(t)+\int_{0}^{T} G(t, s) h(s) d s
$$


where $v$ is the unique solution of the problem

$$
[D u](t)=0, t \in I=[0, T], B(u)=b .
$$

To study the nonlinear problem (2), we now define the superposition operator $N: C(I) \rightarrow C(I)$ by $[N u](t)=f(t, u(t))$. For $u, v \in C(I)$, we write $u \leqslant v$ on $I$ if $u(t) \leqslant v(t)$ for every $t \in I$. If $u \leqslant v$ on $I$, we define the sector

$$
[u, v]=\{w \in C(I): u \leqslant w \leqslant v \text { on } I\} .
$$

We say that a function $\alpha \in C^{n}(I)$ is a lower solution of (2) if

$$
D \alpha \leqslant N \alpha \text { on } I, B(\alpha) \leqslant b .
$$

Analogously, we say that $\beta \in C^{n}(I)$ is an upper solution of (2) if

$$
D \beta \geqslant N \beta \text { on } I, B(\beta) \leqslant b .
$$

To develop the upper and lower solution method, it is necessary to have at our disposal a maximum principle. For instance, in some cases we know that

$$
D u \geqslant 0, B(u) \geqslant 0 \Longrightarrow u \geqslant 0 .
$$

Note that if this maximum principle is valid, then (4) is uniquely solvable for any $h \in C(I)$. Moreover, $G \geqslant 0$ on $I \times I$.

Now, let $\alpha$ be a lower solution and $\beta$ be an upper solution of (2) respectively. Roughly speaking, it is possible to prove that if $\alpha \leqslant \beta$, then there exists a solution $u$ of (2) between the lower and upper solutions. Indeed, in many cases we have that

$$
\alpha \leqslant \beta \Longrightarrow \text { there exists } u \in[\alpha, \beta] \text {, a solution of (2). }
$$

For several particular cases, see [3].

We note that if (8) holds and $f$ satisfies a growth condition, then it possible to generate monotone sequences converging to solutions of the problem.

TheOREM 2.1. (Monotone Iterative Technique.) Assume that (8) is satisfied and let $\alpha$ be a lower solution and $\beta$ be an upper solution of (2) respectively. If there exists $K>0$ such that for every $t \in I$ and $u, v \in \mathbb{R}$ with $\alpha(t) \leqslant v \leqslant u \leqslant \beta(t)$ we have

$$
f(t, u)-f(t, v) \geqslant-K(u-v),
$$

then, there exist monotone sequences $\left\{\alpha_{n}\right\} \nearrow \rho,\left\{\beta_{n}\right\} \searrow r$ uniformly on $I$. Here, $\rho$ and $r$ are the minimal and maximal solutions of (2) respectively between $\alpha$ and $\beta$.

For a proof of this result, see [3]. 


\section{Main Result}

In order to develop the quasilinearisation method, we shall suppose that $\alpha$ and $\beta$ are lower and upper solutions of (2) respectively with $\alpha \leqslant \beta$ on $I$. Let

$$
\Omega=\{(t, u) \in I \times \mathbb{R}: \alpha(t) \leqslant u \leqslant \beta(t)\} .
$$

We introduce the following conditions:

$$
\begin{gathered}
f_{u}(t, u), f_{u u}(t, u) \text { exist and are continuous for }(t, u) \in \Omega, \\
f_{u u}(t, u) \geqslant-2 M, M>0, \text { for }(t, u) \in \Omega . \\
f_{u}(t, u) \leqslant 0 \text { for }(t, u) \in \Omega
\end{gathered}
$$

If condition (11) holds, then for $u$, $v$ with $\alpha(t) \leqslant v \leqslant u \leqslant \beta(t)$, define the function

$$
g(t, u, v)=f(t, v)+\left[f_{u}(t, v)+2 M v\right](u-v)-M\left(u^{2}-v^{2}\right) .
$$

Observe that $g(t, u, u)=f(t, u)$, and that if we define

$$
F(t, u)=f(t, u)+M u^{2},
$$

then condition (12) means that $F_{u u}(t, u) \geqslant 0$ for $(t, u) \in \Omega$.

Note that if $f$ satisfies (11) and (12), then, for any $u, v$ such that $\alpha(t) \leqslant v \leqslant u \leqslant$ $\beta(t)$, we have that

$$
f(t, u) \geqslant g(t, u, v)
$$

Lemma 3.1. Assume that (8) and (9) hold. Consider the nonlinear problem

$$
D u=N_{0} u \text { on } I, B(u)=b
$$

where $\left[N_{0} u\right](t)=g(t, u(t), \alpha(t))$. It admits at least one solution $u \in[\alpha, \beta]$.

Proof: We have that

$$
D \alpha \leqslant N \alpha=N_{0} \alpha \text { on } I, B(\alpha) \leqslant b,
$$

and

$$
D \beta \geqslant N \beta \geqslant N_{0} \beta \text { on } I, B(\beta) \geqslant b .
$$

Hence, $\alpha$ and $\beta$ are lower and upper solutions of (16) respectively. By (9) we have that there exists a solution $u \in[\alpha, \beta]$ of (16). 
THEOREM 3.1. Assume that (8) and (9) hold and and that $f$ satisfies (11), (12) and (13). If $w_{0} \leqslant \gamma$ are lower and upper solutions of (2) respectively, then there exist $w_{k} \in C^{n}(I), k=0,1,2, \ldots$ such that the sequence $\left\{w_{k}\right\}$ is monotone increasing and it converges uniformly and quadratically to a solution $w$ of (2).

Proof: By Lemma 3.1 we know that there exists $w_{1} \in\left[w_{0}, \gamma\right]$, a solution of (16). Thus, we assume that we have constructed $w_{k}, k \geqslant 1$ such that $w_{0} \leqslant w_{1} \leqslant \ldots w_{k} \leqslant \gamma$. Using $w_{k}$ we consider the following nonlinear boundary value problem:

$$
D w=N_{k} w \text { on } I, B(w)=b
$$

where $N_{k}$ is the operator generated by the function $g_{k}: I \times \mathbb{R} \rightarrow \mathbb{R}, g_{k}(t, w)=$ $g\left(t, w, w_{k}(t)\right)$. Thus,

$$
\left[N_{k} w\right](t)=g_{k}(t, w(t))=g\left(t, w(t), w_{k}(t)\right), t \in I
$$

We have that

$$
D w_{k}=N_{k-1} w_{k-1} \leqslant N w_{k}=N_{k} w_{k} \text { on } I, B\left(w_{k}\right) \leqslant b
$$

and

$$
D \gamma \geqslant N \gamma \geqslant N_{k} \gamma \text { on } I, B(\gamma) \geqslant b,
$$

that is, $w_{k}$ and $\gamma$ are lower and upper solutions for (17), respectively. By property (9) we have that there exists $w_{k+1} \in\left[w_{k}, \gamma\right]$, a solution of (17). Hence, we have constructed a sequence $\left\{w_{k}\right\}$ with $w_{0} \leqslant w_{1} \leqslant \ldots \leqslant w_{k} \leqslant w_{k+1} \leqslant \ldots \leqslant \gamma$. Let $w$ be the pointwise limit of $\left\{w_{k}\right\}$.

We now show that $w$ is a solution of (2). Indeed, by (8), we have the following integral representation for $w_{k+1}$

$$
w_{k+1}(t)=v(t)+\int_{0}^{T} G(t, s) g\left(s, w_{k+1}(s), w_{k}(s)\right) d s
$$

Therefore, $\left\{w_{k}\right\}$ is bounded in $C^{n}(I), n \geqslant 1$ and $\left\{w_{k}\right\} \rightarrow w$ uniformly on $I$. Passing to the limit, when $k \rightarrow \infty$, in (18) we obtain that

$$
w(t)=v(t)+\int_{0}^{T} G(t, s) g(s, w(s), w(s)) d s=\int_{0}^{T} G(t, s) f(s, w(s)) d s
$$

and $w$ is a solution of (2).

Finally, we show that the convergence is quadratic. Let $e_{k}=w-w_{k} \geqslant 0$ be the 
error when we approximate $w$ by $w_{k}$. Hence, for $k \geqslant 1, t \in I$ we can write

$$
\begin{aligned}
{\left[D e_{k+1}\right](t)=} & {[D w](t)-\left[D w_{k+1}\right](t)=f\left(t, w_{k+1}(t)\right)-g\left(t, w_{k+1}(t), w_{k}(t)\right) } \\
= & f(t, w(t))-f\left(t, w_{k}(t)\right)-F_{u}\left(t, w_{k}(t)\right) \cdot\left[w_{k+1}(t)-w_{k}(t)\right] \\
& \quad+M\left[w_{k+1}^{2}(t)-w_{k}^{2}(t)\right] \\
= & f(t, w(t))-M w_{k}^{2}(t)-f\left(t, w_{k}(t)\right)-F_{u}\left(t, w_{k}(t)\right) \cdot\left[w_{k+1}(t)-w_{k}(t)\right] \\
\quad & \quad+M w_{k+1}^{2}(t) \\
= & F(t, w(t))-F\left(t, w_{k}(t)\right)-F_{u}\left(t, w_{k}(t)\right) \cdot\left[w_{k+1}(t)-w_{k}(t)\right] \\
& \quad-M\left[w^{2}(t)-w_{k+1}^{2}(t)\right] .
\end{aligned}
$$

Now, using the mean value theorem, we have that there exist $\xi=\xi(t)$ and $\eta=\eta(t)$ such that $w_{k}(t) \leqslant \eta \leqslant \xi \leqslant \gamma(t)$ with

$$
\begin{aligned}
{\left[D e_{k+1}\right](t)=} & F_{u}(t, \xi(t)) \cdot\left[w(t)-w_{k}(t)\right]-F_{u}\left(t, w_{k}(t)\right) \cdot\left[w_{k+1}(t)-w(t)\right] \\
& \quad-M\left[w^{2}(t)-w_{k+1}^{2}(t)\right] \\
= & \left.F_{u}(t, \xi(t))-F_{u}\left(t, w_{k}(t)\right)\right] \cdot\left[w(t)-w_{k}(t)\right] \\
\quad & \quad\left[F_{u}\left(t, w_{k}(t)\right)-M\left(w(t)+w_{k+1}(t)\right)\right] \cdot\left[w(t)-w_{k+1}(t)\right] \\
= & F_{u u}(t, \eta(t)) \cdot\left[\xi(t)-w_{k}(t)\right] \cdot\left[w(t)-w_{k}(t)\right] \\
\quad & \quad\left[F_{u}\left(t, w_{k}(t)\right)-M\left(w(t)+w_{k+1}(t)\right)\right] \cdot\left[w(t)-w_{k+1}(t)\right] .
\end{aligned}
$$

Therefore, $e_{k+1}$ is solution of the following boundary value problem:

$$
D e_{k+1}=\phi_{k}+\psi_{k} \cdot e_{k+1}, B\left(e_{k+1}\right)=0,
$$

where

$$
\phi_{k}(t)=F_{u u}(t, \eta(t)) \cdot\left[\xi(t)-w_{k}(t)\right] \cdot\left[w(t)-w_{k}(t)\right]
$$

and

$$
\psi_{k}(t)=F_{u}\left(t, w_{k}(t)\right)-M\left(w(t)+w_{k+1}(t)\right) .
$$

Hence, we have that

$$
e_{k+1}(t)=\int_{0}^{T} G(t, s) \cdot\left[\phi_{k}(s)+\psi_{k}(s) \cdot e_{k+1}(s)\right] d s .
$$

Now, let $c=\operatorname{Sup}\left\{F_{u u}(t, w):(t, w) \in \Omega\right\}$ and $\lambda=c \cdot \max _{t \in I} \int_{0}^{T} G(t, s) d s$. Denote by \|\|$_{\infty}$ the uniform norm in $C(I)$. Taking into account (13), we have that

$$
0 \leqslant e_{k+1}(t) \leqslant \int_{0}^{T} G(t, s) \phi_{k}(s) d s \leqslant c \int_{0}^{T} G(t, s) e_{k}^{2}(s) d s \leqslant \lambda \cdot\left\|e_{k}\right\|_{\infty}^{2} .
$$

In consequence,

$$
\left\|e_{k+1}\right\|_{\infty} \leqslant \lambda \cdot\left\|e_{k}\right\|_{\infty}^{2}
$$

and the convergence is quadratic. 


\section{EXAMPLES}

We now present some examples to illustrate the main result given in Theorem 3.1. EXAMPLE 1. Consider the initial value problem for a first order ordinary differential equation, that is,

$$
D u=u^{\prime}
$$

and

$$
B(u)=u(0)=u_{0} \in \mathbb{R} .
$$

Then, it is clear that the maximum principle (8) holds in this case since $u^{\prime} \geqslant 0$ on $I$ and $u(0) \geqslant 0$ implies that $u \geqslant 0$ on $I$. Moreover, the existence of a lower solution $\alpha$ and an upper solution $\beta$ with $\alpha \leqslant \beta$ implies the existence of at least one solution $u \in[\alpha, \beta]$. See, for instance, $[3]$.

Therefore, under the conditions of Theorem 3.1 we obtain the existence of a monotone sequence of approximate solutions that converge uniformly and quadratically to a solution. This is precisely the result obtained in [4].

EXAMPLE 2. We now examine the periodic boundary value problem for a first order ordinary differential equation. Indeed, let

$$
D u=u^{\prime}+\lambda u, \lambda>0
$$

and

$$
B(u)=u(0)-u(T)=0 .
$$

In this case the maximum principle (8) holds [3], and the upper and lower solution property (9) is valid [3]. Thus, we obtain the quasilinearisation method for the periodic boundary value problem for a first order ordinary differential equation as in $[5,6]$.

Example 3. Now, let

$$
D u=-u^{\prime \prime}
$$

and

$$
B(u)=\left(v_{0} u(0)-w_{0} u^{\prime}(0), v_{T} u(T)+w_{T} u^{\prime}(T)\right)=\left(b_{0}, b_{1}\right)
$$

with $v_{0}, v_{T} \geqslant 0, v_{0}+v_{T}>0, w_{0}>0$, and $w_{T}>0$. For this boundary value problem for a second order ordinary differential equation we have again (see [3]) the validity of (8) and (9). In consequence, under suitable conditions, we can construct a sequence of approximate solutions converging quadratically to a solution of the problem. This was recently proved in [8]. 
EXAMPLE 4. Finally, we deal with the periodic boundary value problem for an ordinary differential equation of higher order. Let

$$
D u=u^{(n)}+\lambda u, \lambda>0
$$

and

$$
B(u)=\left(u^{(i)}(0)-u^{(i)}(T)\right)_{i=0}^{n-1}=0
$$

By Theorem 4 in [7] we have that for

$$
\lambda<\frac{n^{n} \cdot n !}{[n / 2]^{2} \cdot T^{n} \cdot(n-1)^{n-1}}
$$

$D u \geqslant 0$ and $B(u)=0$ implies that $u \geqslant 0$ on $I$. Also, by the results of [7], we have that (9) is valid and we conclude that the quasilinearisation technique is applicable to this higher order problem.

\section{REFERENCES}

[1] R. Bellman, Methods of nonlinear analysis, Vol. II (Academic Press, New York, 1973).

[2] R. Bellman and R. Kalaba, Quasilinearization and nonlinear boundary value problems (American Elsevier, New York, 1965).

[3] G.S. Ladde, V. Lakshmikantham and A.S. Vatsala, Monotone iterative techniques for nonlinear differential equations (Pitman, Boston, 1985).

[4] V. Lakshmikantham and S. Malek, 'Generalized quasilinearization', Nonlinear World 1 (1994), 59-63.

[5] V. Lakshmikantham and J.J. Nieto, 'Generalized quasilinearization for nonlinear first order ordinary differential equations', Nonlinear Times and Digest 2 (1995), 1-10.

[6] V. Lakshmikantham, N. Shahzad and J.J. Nieto, 'Methods of generalized quasilinearization for periodic boundary value problems', Nonlinear Anal. 27 (1966), 143-151.

[7] V. Seda, J.J. Nieto and M. Gera, 'Periodic boundary value problems for nonlinear higher order ordinary differential equations', Appl. Math. Comput. 48 (1992), 71-82.

[8] N. Shahzad and A.S. Vatsala, 'Improved generalized quasilinearization method for second order boundary value problem', Dynamic Systems Appl. 4 (1995), 79-85.

[9] I. Stakgold, Green's functions and boundary value problems (John Wiley, New York, 1980).

Departamento de Análisis Matemático

Facultad de Matemáticas

Universidad de Santiago de Compostela

15706 Spain

email: amnieto@usc.es 